\title{
Competing Misfit Relaxation Mechanisms in Epitaxial Correlated Oxides
}

\author{
Felip Sandiumenge, ${ }^{1, *}$ José Santiso, ${ }^{2}$ Lluís Balcells, ${ }^{1}$ Zorica Konstantinovic, ${ }^{1}$ Jaume Roqueta, ${ }^{2}$ Alberto Pomar, ${ }^{1}$ \\ Juan Pedro Espinós, ${ }^{3}$ and Benjamín Martínez ${ }^{1}$ \\ ${ }^{1}$ Institut de Ciència de Materials de Barcelona, CSIC, Campus de la Universitat Autònoma de Barcelona, \\ E-08193 Bellaterra, Catalonia, Spain \\ ${ }^{2}$ Centre for Nanoscience and Nanotechnology, CIN2 (CSIC-ICN), Campus de la Universitat Autònoma de Barcelona, \\ E-08193 Bellaterra, Catalonia, Spain \\ ${ }^{3}$ Materials Science Institute of Seville, ICMS (CSIC-University of Seville), c/Américo Vespucio 49, E-41092 Seville, Spain
} (Received 9 May 2012; revised manuscript received 22 December 2012; published 8 March 2013)

\begin{abstract}
Strain engineering of functional properties in epitaxial thin films of strongly correlated oxides exhibiting octahedral-framework structures is hindered by the lack of adequate misfit relaxation models. Here we present unreported experimental evidence of a four-stage hierarchical development of octahedralframework perturbations resulting from a progressive imbalance between electronic, elastic, and octahedral tilting energies in $\mathrm{La}_{0.7} \mathrm{Sr}_{0.3} \mathrm{MnO}_{3}$ epitaxial thin films grown on $\mathrm{SrTiO}_{3}$ substrates. Electronic softening of the $\mathrm{Mn}-\mathrm{O}$ bonds near the substrate leads to the formation of an interfacial layer clamped to the substrate with strongly degraded magnetotransport properties, i.e., the so-called dead layer, while rigid octahedral tilts become relevant at advanced growth stages without significant effects on charge transport and magnetic ordering.
\end{abstract}

DOI: 10.1103/PhysRevLett.110.107206

Thin film epitaxy provides the common playground for tailoring materials functionalities through misfit strain. However, notwithstanding the huge attention focused on this issue $[1,2]$, the lack of understanding of misfit relaxation mechanisms in octahedral framework structures still constitutes a serious drawback for a deterministic manipulation of the lattice, electronic, and magnetic degrees of freedom which characterize them. Controlling octahedral tilts and distortions in perovskite-type transition-metal oxides has accordingly emerged as a critical step towards exploiting their unique capabilities for electronic and spintronic applications [3]. Notably, epitaxial perovskites often bypass misfit-dislocation mediated mechanisms that successfully describe the relaxation behavior of semiconducting epitaxial films [4]. Examples are found among a variety of functional films like ferroelectric $\mathrm{PbTiO}_{3}$ [5], multiferroic $\mathrm{BiFeO}_{3}$ [6], and $\mathrm{TbMnO}_{3}$ [7], where misfit strains are relieved by a combination of symmetry changes and ferroelastic domains. Special interest is being attracted by the room temperature $\left(T_{C} \sim 370 \mathrm{~K}\right)$ half-metal ferromagnet $\mathrm{La}_{0.7} \mathrm{Sr}_{0.3} \mathrm{MnO}_{3}$ (LSMO) [3]. The intriguing degradation of its magnetotransport performance near the interface, constitutes, however, a serious drawback whose microscopic origin remains obscure. Prior reports converge in signaling a $\mathrm{Mn}^{3+}$ enrichment near the interface $[8,9]$ and a preferential occupation of $d-e_{g} 3 z^{2}-r^{2}$ orbitals [10-12], leading to a local $C$-type antiferromagnetic ordering. Disentangling the effects of the various constraints imposed by the interface (symmetry breaking [13], elastic strain [12], interaction between the $\mathrm{Mn}$ and Ti electronic structures [12], and polar discontinuity [14]) thus emerges as a cornerstone for the understanding of misfit relaxation
PACS numbers: 75.47.Lx, 68.55.-a, 75.30.Kz, 75.70.Cn

mechanisms in correlated-oxide epitaxial framework structures.

To explain their elastic behavior, here we consider that the deformation of perovskite-type $\mathrm{ABO}_{3}$ octahedral frameworks is governed by the relative strength between the $B-\mathrm{O}-\mathrm{B}$ bond angles bridging adjacent $\mathrm{BO}_{6}$ octahedra and the $B$-O bonds. Strain may couple with octahedral tilts $[15,16]$, or with $B-\mathrm{O}$ bonds either at the expense of elastic or electronic energies. Strongly correlated oxides constitute an ideal arena to explore the effects of biaxial strain on the delicate balance between lattice, spin, charge, and orbital degrees of freedom [17]. To address this issue, here we investigate the thickness dependence of lattice distortions in $\mathrm{La}_{0.7} \mathrm{Sr}_{0.3} \mathrm{MnO}_{3}$ films grown on (001)- $\mathrm{SrTiO}_{3}$ (STO) substrates.

Films with thicknesses ranging between 1.9 and $475 \mathrm{~nm}$ were grown by rf magnetron sputtering on $\mathrm{TiO}_{2}$ terminated STO substrates [18]. Kinetic conditions were adjusted to guarantee a 2D layer-by-layer growth mode and avoid growth induced deterioration of the films [19]. The strain state was investigated by $\mathrm{x}$-ray diffraction determinations of the out-of-plane $(c)$ and in-plane $(a)$ lattice parameters by fitting positions of both integer and half-order superstructure reflections in order to account for interferences between substrate and film reflections in the low thickness range $(<4 \mathrm{~nm})$ [19]. The 3D shear strain state of the films was determined by measuring the in-plane $\left(\chi^{\|}\right)$and out-of-plane $\left(\chi^{\perp}\right)$ components of the shear angle, $\chi_{i}=$ $\alpha_{\mathrm{rh}, i}-90^{\circ}$ [19]. Twin patterns were directly observed by orientation contrast electron backscattered (OC-EBS) images obtained in a field emission scanning electron microscope. The magnetic and charge transport properties of the 
films were studied between 10 and $380 \mathrm{~K}$. The in-plane electrical resistivity was measured using the standard fourpoint geometry with a constant applied current of $5 \mathrm{nA}$. Magnetization measurements were performed using a SQUID magnetometer under perpendicular applied field of $5 \mathrm{kOe}$. The Mn oxidation state was explored by means of $\mathrm{x}$-ray photoemission spectroscopy (XPS).

The evolution of the lattice parameters with increasing film thickness is shown in Fig. 1(a). A progressive decrease of the $c$-axis parameter from the thinnest film $(h \sim 1.9 \mathrm{~nm})$ down to a minimum value at thicknesses comprised between 10 and $25 \mathrm{~nm}$ followed by a smooth increase is clearly seen. This result agrees with a dilation of the substrate-film interface, as reported by other authors [20]. Notably, this trajectory is accompanied by a constant inplane fully strained $a$-axis parameter throughout the whole thickness range. In the full thickness range $1 / 21 / 2 L$ crystal truncation rod exploration has shown half-order reflections at $L=3 / 2$ and $5 / 2$ (absence at $L=1 / 2$ )
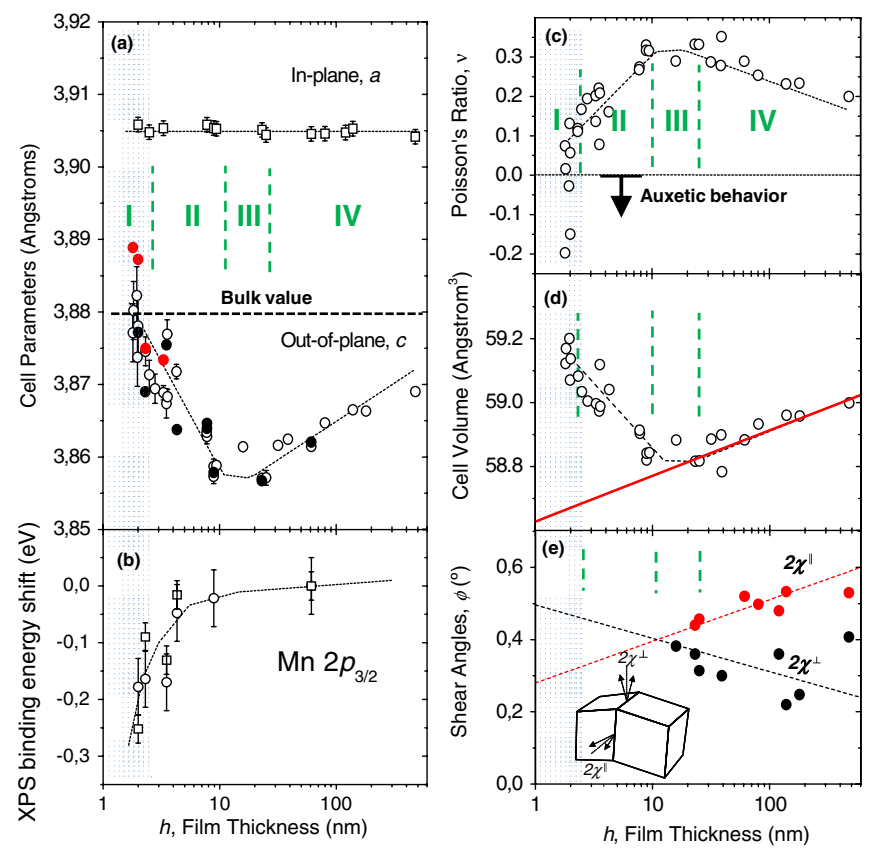

FIG. 1 (color online). (a) Thickness dependence of the inplane and out-of-plane lattice parameters. For $c$-axis parameters, open symbols were obtained by fitting 002 rocking profiles, black closed symbols from 1/2 1/2 3/2 superstructure reflections, and red symbols (gray) by averaging over 17 different $h / 2$ $k / 2 l / 2(h, k, l=1,3,5)$ superstructure reflections [19]. (b) Shift in the XPS binding energies of the Mn $2 p_{3 / 2}$ peak for LSMO films with different thicknesses, relative to the $61 \mathrm{~nm}$ thick sample. Circles and squares correspond to measurements performed with Escalab and Phoibos equipments, respectively, [19]. (c) Thickness dependence of the Poisson's ratio. (d) Thickness dependence of the pseudo cubic unit cell volume. (e) Thickness dependence of the in-plane $\left(\chi^{\|}\right)$and out-of-plane $\left(\chi^{\perp}\right)$ shear angles. The shadowed area indicates the nontwinned thickness range. consistent with rhombohedral $a^{-} a^{-} a^{-}(R-3 c)$, orthorhombic $a^{-} a^{-} c^{0}$ (Imma), and monoclinic $a^{-} a^{-} c^{-}(C 2 / c)$ tilt systems [21]. According to this evolution, we identify in Fig. 1(a) four different deformation regimes: namely, (I) for $h<2.5 \mathrm{~nm}$, (II) for $2.5 \mathrm{~nm}<h<10 \mathrm{~nm}$, (III) for $10 \mathrm{~nm}>h>25$, and (IV) for $h>25 \mathrm{~nm}$ up to $475 \mathrm{~nm}$. As depicted in Fig. 1(b), XPS of the Mn $2 p 3 / 2$ peak shows a progressive shift of $-0.2 \mathrm{eV}$ towards lower binding energies as the film thickness is decreased below $10 \mathrm{~nm}$, particularly within regime I. According to Abbate et al. [22], this shift would correspond to a variation of 0.18 in the oxidation state of Mn which would drive the LSMO phase into a nonferromagnetic and insulating state [23].

The resulting evolution of the Poisson's ratio, $(\nu=$ $\varepsilon^{\perp} /\left(\varepsilon^{\perp}-2 \varepsilon^{\|}\right)$, where $\varepsilon^{\perp}$ and $\varepsilon^{\|}$are the out-of-plane and in-plane strain components ( $a=3.881 \AA$ [24] is taken as a reference value), is shown in Fig. 1(c), and the evolution of the unit cell volume, $V(h)$, is depicted in Fig. 1(d). The shadowed area, where films exhibit auxetic behavior, indicates the nontwinned thickness range (see below). In regime III, $\nu \sim 0.33$, is the closest value to that derived from the elastic constants determined for a $\mathrm{La}_{0.83} \mathrm{Sr}_{0.17} \mathrm{MnO}_{3}$ single crystal, $\nu=0.41[19,25]$.

Concomitantly, the in-plane $\left(\chi^{\|}\right)$and out-of-plane $\left(\chi^{\perp}\right)$ components of the rhombohedral shear, $\alpha_{\mathrm{rh}}-90^{\circ}$, also converge in regime III [Fig. 1(e)] (recall that the rhombohedral structure imposes $\chi^{\|}=\chi^{\perp}$ ). Therefore, regime III corresponds to a pure elastically strained state of the rhombohedral LSMO film without invoking any significant octahedral tilting perturbation but a compression of the $\mathrm{MnO}_{6}$ octahedra along the $c$ axis, in agreement with previous spectroscopic analyses [26]. In order to correlate the divergence between $\chi^{\|}$and $\chi^{\perp}$ observed for $h>10 \mathrm{~nm}$ with the perturbation of the octahedral tilt pattern, here we use the formalism developed for rhombohedral perovskites [27] to derive an approximate analytical expression for the unit cell volume dependence on the in-plane, $\alpha(=\beta)$, and out-of-plane, $\gamma$, octahedral tilt angles about the $[100] /[010]$ and [001] axes, respectively: $V \approx$ $a_{0}{ }^{3}\left(1-\gamma^{2}\right)\left(1-\alpha^{2}\right)^{2}$, where $a_{0}$ is the equilibrium lattice parameter of the undistorted cubic prototype [19]. The resulting $V(h)$ dependence shows an excellent agreement with that calculated from the lattice parameters, as illustrated by a red solid line in Fig. 1(d). This indicates that the relaxation pathway followed in regime IV is mediated by a progressive decrease of $\alpha(=\beta)$, causing a simultaneous enlargement of the three principal axes of the unit cell, accompanied by a increase of $\gamma$ which has no effect on the $c$-axis parameter but compensates the effect on $a$ (and $b$ ), thus preserving the in-plane matching with the substrate.

Turning now our attention to the very early growth stages, we illustrate in Fig. 2 the evolution of the shear strain state through film thicknesses of $1.9 \mathrm{~nm}$ (a) and $3.3 \mathrm{~nm}$ (b). The twin pattern is recognized in the OC-EBS image shown in Fig. 2(b), left panel, as a 

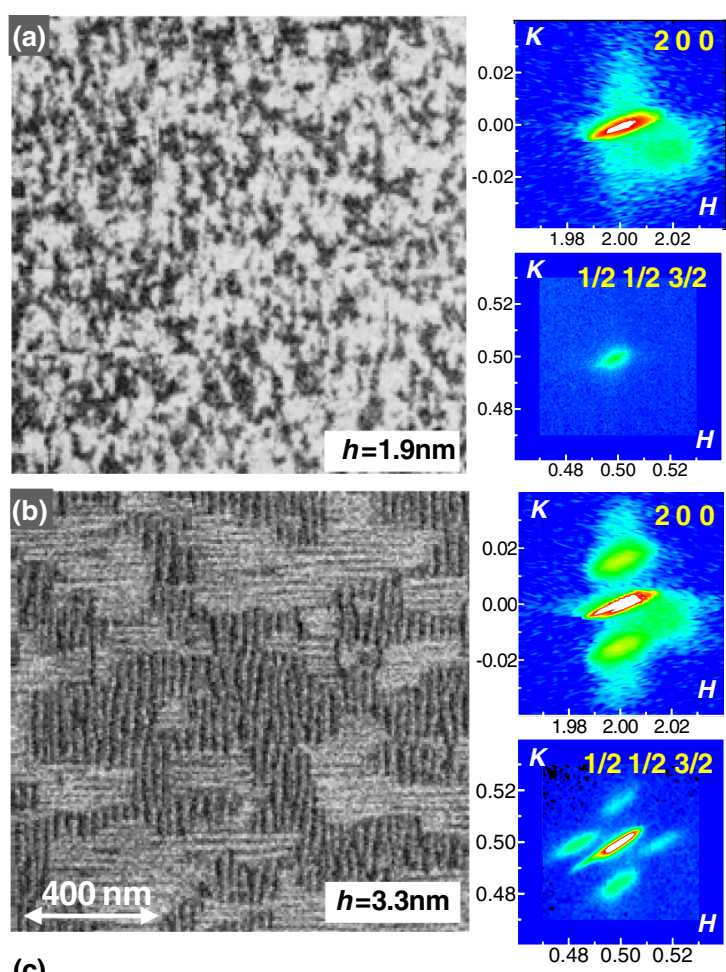

(c)
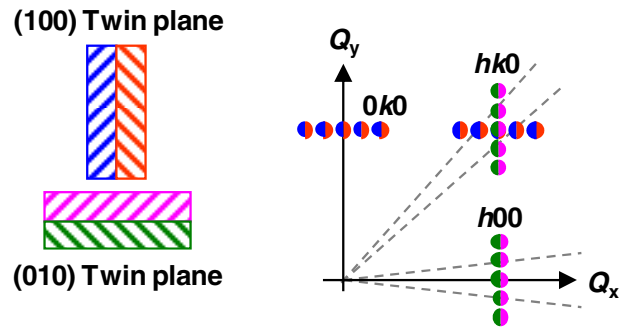

FIG. 2 (color online). OC-EBS images corresponding to $1.9 \mathrm{~nm}$ (a) and $3.3 \mathrm{~nm}$ (b) thick films. Right panels show the corresponding $H K$ RSM's of the 200 and 1/2 1/2 3/2 reflections. (c) Schematics showing the effect of (100) and (010) twin planes on diffraction.

patchwork of twin families seen as parallel stripes of alternating dark or bright contrast aligned with the [100] and [010] film directions. Conversely, in the $1.9 \mathrm{~nm}$ film shown in Fig. 2(a), this contrast is absent. The speckled contrast is attributed to the coexistence of two degenerated monoclinic epitaxial orientations. The corresponding $H K$ reciprocal space maps (RSM's) around the film and substrate 200 and film 1/2 1/2 3/2 reflections are shown in the right panels. For the $1.9 \mathrm{~nm}$ thick film, neither the 200 nor the $1 / 21 / 23 / 2$ reflections exhibit any signature of twinning. In the thicker film, the diffuse scattering associated with the 200 reflection along the $K$ axis defines up to second order modulation satellites signaling the development of the lateral twin periodicity [28]. Moreover, the sharp fourfold splitting along the $[100]^{*}$ and $[010]^{*}$ reciprocal directions observed around the 1/2 1/2 3/2 halforder reflection is consistent with the development of an homogeneous rhombohedral $(100) /(010)$ twin structure as schematically depicted in Fig. 2(c). A similar analysis of a $2.7 \mathrm{~nm}$ thick film (not shown) revealed weaker twin signatures in the RSM's. These observations contrast with equilibrium models of ferroelastic domains in epitaxial LSMO predicting an exponential decay of the twin width with increasing thickness for $h<3 \mathrm{~nm}$ [29], and indicate an spontaneous build up of the shear strain at a critical thickness, $h_{\text {shear }}$, comprised between 2 and $2.5 \mathrm{~nm}$ (5-7 unit cells).
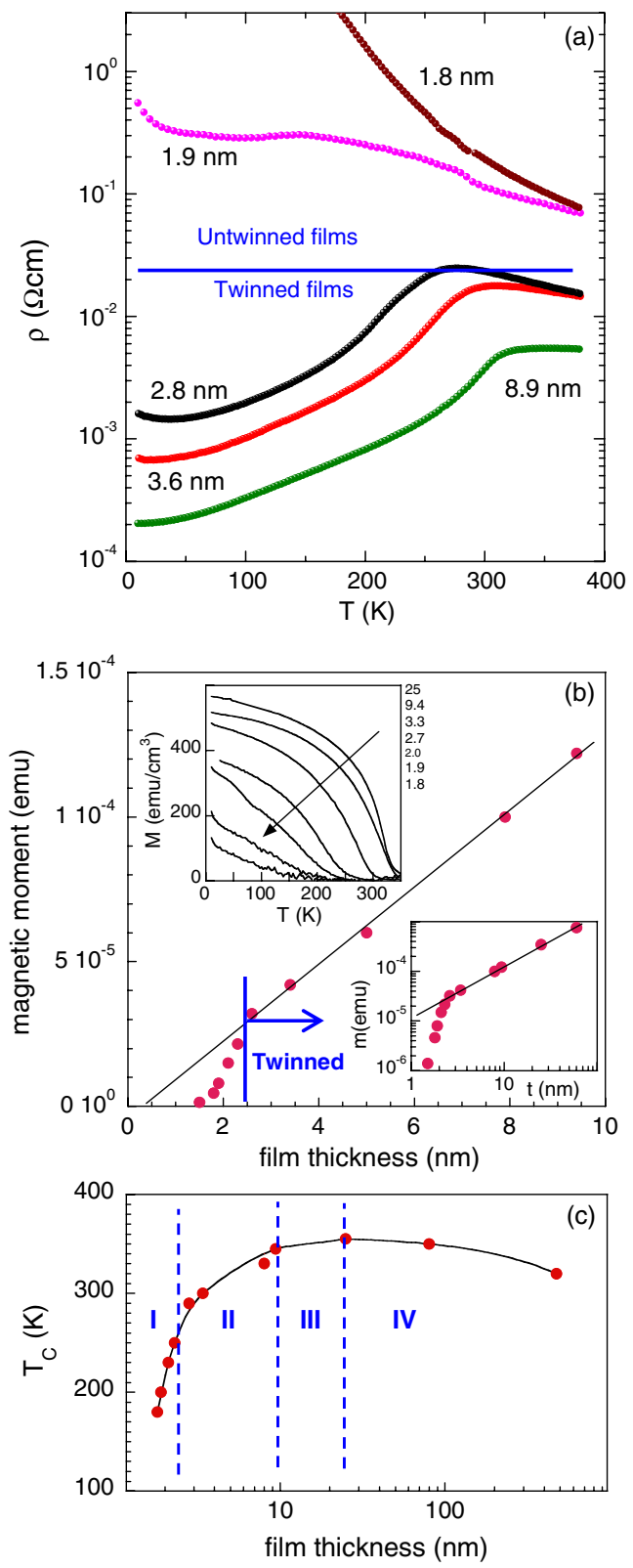

FIG. 3 (color online). (a) Resistivity versus temperature curves for films having different thickness above and below the shear transition thickness, as indicated. (b) Thickness dependence of the magnetic moment for $h<10 \mathrm{~nm}$. Lower inset shows the dependence in an expanded thickness range. Upper inset: Temperature dependence of the magnetization at low field $(H=$ $5 \mathrm{kOe}$ ). (c) Thickness dependence of the Curie temperature, $T_{C}$. 
The evolution of the temperature dependence of the resistivity, $\rho(T)$, at these growth stages is shown in Fig. 3(a). It can be seen that for $h<h_{\text {shear }}$, the films are insulating without any signature of insulating-to-metal $(I-M)$ transition between 10 and $380 \mathrm{~K}$. This behavior is accompanied by a sudden drop of the magnetic moment [see Fig. 3(b), main panel and lower inset]. In concomitance with this behavior, the temperature dependence of the magnetization, $M(T)$, shown in the upper inset of Fig. 3(b) also shows that the films are not ferromagnetic (FM). According to our XPS analysis, see Fig. 1(b), this transition is driven by a local increase of the $\mathrm{Mn}^{3+} / \mathrm{Mn}^{4+}$ ratio near the interface, resulting in an enhanced Jahn-Teller distortion and a decrease of the band width. According to the structural $\mathrm{La}_{1-x} \mathrm{Sr}_{x} \mathrm{MnO}_{3}$ phase diagram [30], this effect is analogous to a reduction of the doping level, $x$, which favors the stabilization of monoclinic forms of LSMO. In such a case, the in-plane shear strain is cancelled by placing the monoclinic unique axis out-of-plane. This interpretation agrees with the observation of a critical thickness for twin formation coinciding with the built-up of a shear strain. The value of $h_{\text {shear }} \sim 2.5 \mathrm{~nm}$ coincides with the thickness at which Mn cations start to recover their stoichiometric mean valence, as observed in Fig. 1(b). Moreover, besides the distortion induced by Jahn-Teller and possible $e_{g}\left(3 z^{2}-r^{2}\right)$ orbital reconstruction effects, the mean volume of the $\mathrm{MnO}_{6}$ octahedra is expected to increase as a result of the larger $\mathrm{Mn}^{3+}$ ionic radius $\left(0.645 \AA\right.$ in high-spin state, versus $0.530 \AA$ for $\mathrm{Mn}^{4+}$ [31]). This is clearly manifested by the average increase of the $\mathrm{Mn}-\mathrm{O}$ bond distances in the monoclinic phase of LSMO [30]. This causes an increase of the lattice parameters [30], in excess to that required to fully accommodate the tensile misfit strain with the STO substrate. This scenario explains the observed delay in the formation of an elastically strained rhombohedral phase until the growing film reaches regime III.

Figure 4 shows the thickness dependent phase diagram of LSMO films. The formation of a $\sim 2.5 \mathrm{~nm}$ thick insulating interfacial dead layer (indicated by a shadowed area) lacking FM order is compatible with prior experimental [11] and theoretical [13] reports showing that irrespective of the elastic strain state a preferential occupation of $3 z^{2}-r^{2}$ orbitals is intrinsic to the symmetry breaking at LSMO film surfaces and interfaces. According to theoretical studies, above the shear transition the progressive decay of the $\mathrm{Mn}^{3+}$ concentration would favor the development of inhomogeneous FM patches with variable $\mathrm{Mn}^{3+} / \mathrm{Mn}^{4+}$ ratios [32]. This scenario is consistent with the appearance of the I-M transition in the $\rho(T)$ curves for $h \geq h_{\text {shear }}$ [see Fig. 3(a)], although this occurs at a lower temperature than that corresponding to the bulk Curie temperature $T_{C}$. Regime II, bridging the monoclinic and rhombohedral states, thus defines a transition between the interfacial state and a strained state in which the electronic energy gained by

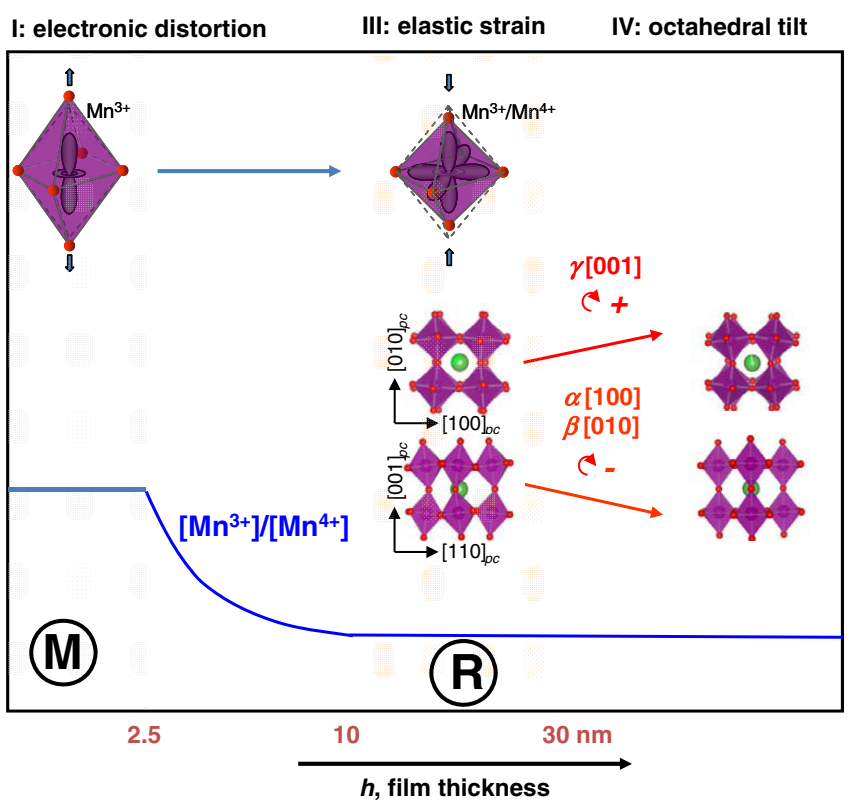

FIG. 4 (color online). Mechanisms of lattice distortions through regimes I,II, III, and IV. Regime II bridging the monoclinic and rhombohedral states, is not labeled in the diagram for clarity. The orbital character and its relation with octahedral strain in the monoclinic $(M)$ and rhombohedral $(R)$ states is indicated. The transition between the two states is mediated by a decrease of the $\mathrm{Mn}^{3+} / \mathrm{Mn}^{4+}$ ratio (blue line). Lower panels illustrate the combined octahedral tilting mechanism releasing the elastic energy stored in regime III.

removing the orbital degeneracy within the $\mathrm{Mn}^{3+} \mathrm{O}_{6}$ coordination environment becomes progressively exceeded by the elastic energy opposing a similar expansion of the equatorial Mn-O distances, until the tensilely strained rhombohedral phase condenses at a thickness of $\sim 10 \mathrm{~nm}$ (onset of regime III). Simultaneously, the typical temperature dependence of the magnetization in a FM material becomes apparent in the $M(T)$ curves shown in the upper inset of Fig. 3(b). Upon increasing the film thickness beyond regime III $(\sim 25 \mathrm{~nm})$, the stored elastic energy is relaxed by a pure octahedral tilting mechanism without detrimental consequences on the metallic and ferromagnetic behavior of the films. The smooth decrease of $T_{C}$ observed at this stage [see Fig. 3(c)] can be attributed to a weakening of the magnetic interactions caused by the tilting perturbation.

In summary, the present work sheds light on the precise role of octahedral tilts and distortions in the misfit-strain relaxation behavior of an epitaxial octahedral-framework correlated-oxide structure. Our results bring up a new misfit relaxation scenario in thin film epitaxy whereby plastic deformation is bypassed by the successive stabilization of characteristic deformation states that satisfactorily explain the intriguing thickness dependence of functional properties observed in LSMO films, including the formation of the so-called interfacial dead layer. 
We thank J. Bassas (SCT, Universitat de Barcelona) and P. Ferrer (European Synchrotron Radiation Facility, ESRFSpline, France) for their valuable support in x-ray diffraction experiments. We acknowledge the Spanish Ministerio de Economia y Competitividad and Consejo Superior de Investigaciones Científicas for financial support and for provision of synchrotron radiation facilities in using beam line BM25-SpLine". This research was supported by Spanish MEC (Grants No. MAT2009-08024 and No. MAT2011-29081), CONSOLIDER (Grants No. CSD2007-00041 and No. CSD2008-00023), and FEDER program. Z. K. thanks the Spanish MEC for the financial support through the $\mathrm{RyC}$ program.

*Corresponding author. felip@icmab.es

[1] J. Chakhalian, A. J. Millis, and J. Rondinelli, Nat. Mater. 11, 92 (2012).

[2] J. He, A. Borisevich, S. V. Kalinin, S. J. Pennycook, and S. T. Pantelides, Phys. Rev. Lett. 105, 227203 (2010).

[3] M. Bibes, J.E. Villegas, and A. Barthélémy, Adv. Phys. 60, 5 (2011).

[4] S. C.Jain, A. H. Harker, and R. A. Cowley, Philos. Mag. A 75, 1461 (1997).

[5] Q. Y. Qiu, V. Nagarajan, and S. P. Alpay, Phys. Rev. B 78, 064117 (2008).

[6] C. J. M. Daumont, S. Farokhipoor, A. Ferri, J. C. Wojdeł, J. Íñiguez, B. J. Kooi, and B. Noheda, Phys. Rev. B 81, 144115 (2010).

[7] C. J. M. Daumont, D. Mannix, S. Venkatesan, G. Catalan, D. Rubi, B. J. Kooi, J. Th. M. De Hosson, and B. Noheda, J. Phys. Condens. Matter 21, 182001 (2009).

[8] T. Riedl, T. Gemming, K. Dörr, M. Luysberg, and K. Wetzig, Microsc. Microanal. 15, 213 (2009).

[9] J.-S. Lee, D. A. Arena, P. Yu, C. S. Nelson, R. Fan, C. J. Kinane, S. Langridge, M. D. Rossell, R. Ramesh, and C.-C. Kao, Phys. Rev. Lett. 105, 257204 (2010).

[10] C. Aruta, G. Ghiringhelli, A. Tebano, N. Boggio, N. Brookes, P. Medaglia, and G. Balestrino, Phys. Rev. B 73, 235121 (2006).

[11] A. Tebano et al., Phys. Rev. Lett. 100, 137401 (2008); 103, 079902(E) (2009).
[12] M.-B. Lepetit, B. Mercey, and Ch. Simon, Phys. Rev. Lett. 108, 087202 (2012).

[13] M. J. Calderón, L. Brey, and F. Guinea, Phys. Rev. B 60, 6698 (1999).

[14] J. L. Maurice, D. Imhoff, J. P. Contour, and C. Colliex, Philos. Mag. A 86, 2127 (2006).

[15] M. T. Dove, M. Gambhir, K. D. Hammonds, and A. K. A. Pryde, Phase Transit. 58, 121 (1996).

[16] A. J. Hatt and N. A. Spaldin, Phys. Rev. B 82, 195402 (2010).

[17] H. Y. Hwang, Y. Iwasa, M. Kawasaki, B. Keimer, N. Nagaosa, and Y. Tokura, Nat. Mater. 11, 103 (2012).

[18] Z. Konstantinovic, J. Santiso, D. Colson, A. Forget, Ll. Balcells, and B. Martinez, J. Appl. Phys. 105, 063919 (2009).

[19] See Supplemental Material at http://link.aps.org/ supplemental/10.1103/PhysRevLett.110.107206 for details on film growth, x-ray diffraction determinations of lattice parameters and shear strains, and a discussion on the discrepancy between Poisson's ratios determined for thin films and single crystals, as well as for XPS measurement details.

[20] R. Herger et al., Phys. Rev. B 77, 085401 (2008).

[21] A. M. Glazer, Acta Crystallogr. Sect. A 31, 756 (1975).

[22] M. Abbate et al., Phys. Rev. B 46, 4511 (1992).

[23] B. Dabrowski et al., Phys. Rev. B 60, 7006 (1999).

[24] P. Radaelli, G. Iannone, M. Marezio, H. Hwang, S.-W. Cheong, J. Jorgensen, and D. Argyriou, Phys. Rev. B 56, 8265 (1997).

[25] T. W. Darling, A. Migliori, E. G. Moshopoulou, S. A. Trugman, J. J. Neumeier, J. L. Sarrao, A. R. Bishop, and J. D. Thompson, Phys. Rev. B 57, 5093 (1998).

[26] N. M. Souza-Neto, A. Y. Ramos, H. C. N. Tolentino, E. Favre-Nicolin, and L. Ranno, Phys. Rev. B 70, 174451 (2004).

[27] H. D. Megaw and C. N. W. Darlington, Acta Crystallogr. Sect. A 31, 161 (1975).

[28] U. Gebhardt, N. Kasper, A. Vigliante, P. Wochner, H. Dosch, F. Razavi, and H.-U. Habermeier, Phys. Rev. Lett. 98, 096101 (2007).

[29] N. Farag, M. Bobeth, W. Pompe, A. E. Romanov, and J. S. Speck, J. Appl. Phys. 97, 113516 (2005).

[30] J. Mitchell, D. Argyriou, C. Potter, D. Hinks, J. Jorgensen, and S. Bader, Phys. Rev. B 54, 6172 (1996).

[31] R. D. Shannon, Acta Crystallogr. Sect. A 32, 751 (1976).

[32] L. Brey, Phys. Rev. B 75, 104423 (2007). 